\title{
Laser nanofabrication deep inside silicon wafers
}

\author{
Rana Asgari Sabet ${ }^{1,2}$, Aqiq Ishraq ${ }^{1,2}$, Onur Tokel ${ }^{1,2}$ \\ 1. Department of Physics, Bilkent University, 06800 Ankara, Turkey \\ 2. UNAM-National Nanotechnology Research Center and Institute of Material Science and Nanotechnology, Bilkent University, 06800 Ankara, Turkey
}

Abstract: Here, we introduce the first controlled nanofabrication capability in the bulk of silicon wafers. We exploit smart use of Bessel beams and demonstrate "in-chip" nano-structuring with features lower than $250 \mathrm{~nm}$.

Silicon is at the heart of electronics and silicon-photonics. These industries are limited to creating functionalities on the wafer surface, which may be broadly classified as "on-chip". However, at the current state, much of the bulk (or subsurface) of the wafer is essentially wasted. Recently, we introduced a new fabrication approach, enabling "in-chip" capabilities, where we showed that infrared laser pulses can realize controlled 3-D modifications deep inside si, without damaging the wafer above or below modifications [1]. We further showed these can be used for introducing advanced functionality into the wafer, e.g., waveguides or holograms, among others [1,2]. A critical milestone would be to tackle the resolution limit of fabrication, that is to overcome the state-of-the-art 1- $\mu$ m resolution limit [1].

Fig. 1. a) Experimental characterization of the Bessel beam used for in-chip nanostructuring. (b) Experimental and theoretical transverse beam profiles. (c) Selective etching reveals nanostructures that formed with features of $\mathbf{2 5 0} \mathbf{~ n m}$. (d) Histogram of the feature sizes of in-chip nano-structures.

Here, we demonstrate the first controlled nano-fabrication

capability deep inside silicon wafers (Fig.1). We exploit a spatially structured laser beam (Fig 1a - 1b), created with a spatial light modulator (SLM). With b

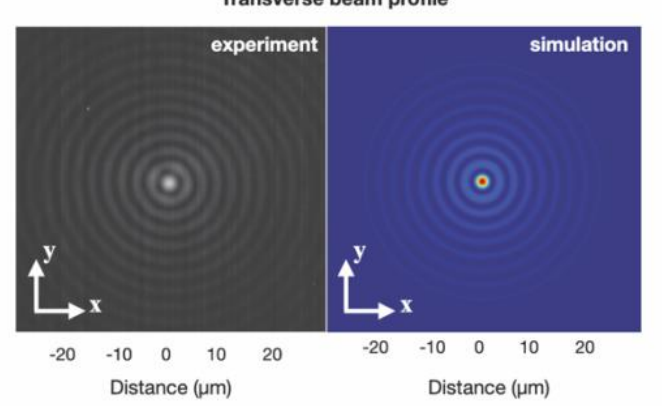

d

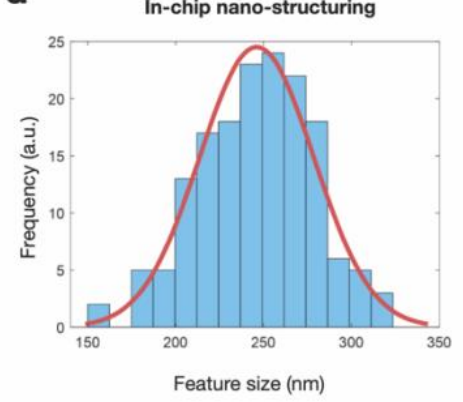

the smart use of spatial beam profiling, we achieve creation of controlled subsurface (in-chip) structures. We observe modification size and length can be controlled by changing the beam profile and laser writing conditions. We show formation of $250 \mathrm{~nm}$-thick in-chip structures, elongating $>100 \mu \mathrm{m}$ along laser propagation direction, reaching aspect ratios $>100$ (Fig. 1c-d). Using different type beams and laser parameters, we further create in-chip structures with features lower than $250 \mathrm{~nm}$. To the best of our knowledge this constitutes the first controlled nanofabrication capability inside silicon. These may be considered analogous to the broader approaches in nano-photonics [3].

\section{References}

[1] O. Tokel, A. Turnali, G. Makey, P. Elahi, T. Colakoglu, E. Ergecen, O. Yavuz, R. Hubner, M. Z. Borra, A. Bek, R. Turan, D. K. Kesim, S. Tozburun, S. Ilday and F. Ö. Ilday, "In-chip microstructures and photonic devices fabricated by nonlinear laser lithography deep inside silicon," Nature Photonics, 11, 639, (2017)

[2] A. Turnali, M. Han, O. Tokel, "Laser-written depressed-cladding waveguides deep inside bulk silicon”, J. Opt. Soc. B., 36, 4, 966 (2019).

[3] A. Ródenas et al., "Three-dimensional femtosecond laser nanolithography of crystals", Nature Photonics, 13, 105, (2019). 